\title{
Short segment coronary artery bypass grafting using radial artery to repair bilateral coronary ostial aneurysms after aortic root replacement in a patient with Marfan syndrome
}

\author{
Kosuke Saku ${ }^{1}$, Kazuyoshi Takagi ${ }^{1}$, Tomofumi Fukuda ${ }^{1}$, Eiki Tayama ${ }^{1}$, and Hiroyuki \\ Tanaka $^{1}$ \\ ${ }^{1}$ Kurume University Hospital
}

January 5, 2021

\begin{abstract}
Coronary ostial aneurysm is one of the complications after aortic root replacement especially in Marfan syndrome. How to reconstruct the coronary arteries is important problems during reoperation. Herein, we report a case of coronary artery bypass grafting using radial artery to repair bilateral coronary ostial aneurysms after aortic root replacement in a patient with Marfan syndrome.
\end{abstract}

\section{Introduction}

Coronary ostial aneurysm (COA) is one of the fatal complications after aortic root replacement (ARR) ${ }^{1,2}$ and the higher incidence in patients with Marfan syndrome (MFS) has been reported. ${ }^{3}$ Surgical procedures to reconstruct coronary ostium is still an important issue to secure coronary perfusion and avoid COA recurrent. Herein, we report a case of short segment coronary artery bypass grafting (CABG) using radial artery (RA) to repair bilateral COA after ARR in a 48-year-old patient with MFS.

\section{Case Report}

A 48-year-old-man with MFS underwent ARR using a mechanical valve and a dacron graft for type A acute aortic dissection. Accidentally bilateral COA (left: $27 \times 21 \mathrm{~mm}$, right: $19 \times 17 \mathrm{~mm}$ ) was found by a computed tomography $(\mathrm{CT})$ at 13 years after previous surgery. We decided to perform re-operation because bilateral coronary ostial aneurysm enlarged by $5 \mathrm{~mm}$ and over per year (left: $32 \times 26 \mathrm{~mm}$, right: $25 \times 21 \mathrm{~mm}$ ) without any symptoms (Figure 1).

Re-median sternotomy was performed. Cardiopulmonary bypass was established between the left common femoral artery and right atrium. Whole tissue around dacron graft including coronary ostium were severely adhered. We could not employ the anatomical reconstruction of coronary ostium using button technique because the remnant of coronary button was very fragile to make coronary button for anastomosis. We decided to perform the short segment CABG using RA graft to the left main trunk (LMT) and right coronary artery (RCA). The LMT, left anterior descending artery, left circumflex artery and RCA could be identified near the aortic root and isolated with silicone strings respectively. The both coronary ostia were resected from the graft and were directly closed with $5 / 0$ monofilament. The side-to-end distal anastomosis to the LMT and RCA using RA were performed with $7 / 0$ monofilament and proximal anastomosis to the aortic root were performed with $6 / 0$ monofilament.

A postoperative CT showed CABG were patent (Figure 2). The patient was discharged uneventfully on postoperative day 19th. Histopathological examination of the aneurysms showed cystic medial necrosis associated 
with MFS.

\section{Discussion}

COA is one of the complications after ARR. ${ }^{1,2}$ Previous studies have reported that COA was frequently seen in patients with MFS. ${ }^{1,2}$

To avoid the recurrence of COA, the reconstruction method of coronary ostium is important. Previous reports have proposed two important points as follows. First, reducing the tension on the anastomosis. ${ }^{4}$ Second, removing the diseased aortic wall around coronary ostium as much as possible. ${ }^{3}$ To reduce the tension, complete resection adhered tissue around coronary button is needed. However, the remnant tissue around coronary ostium often tightly adhered around tissues like this case. Thus, we could not select coronary ostium reconstruction using coronary button technique. Although the graft interposing method like Cabrol's technique are helpful ${ }^{4}$, we need to use the necessary bare minimum of diseased aortic wall around the coronary buttons for anastomosis. Therefore, we considered that the graft interposing technique were not suitable for this patient to prevent the recurrence of COA.

CABG is established and useful technique when we could not employ anatomical reconstruction of coronary ostium. We performed short segment CABG to LMT and proximal RCA using RA, because LMT and proximal RCA could be exposed easily in the same operative field, and it is very easy to harvest the enough length of RA graft for short segment CABG rather than the internal mammary artery during reoperation. In addition, RA graft has not only an enough flow capacity immediately after coronary reconstruction, but also the excellent 20-years patency rate especially when the conduit is used to a vessel with $>90 \%$ stenosis. ${ }^{5}$ The satisfactory patency rate of RA graft was reported similar to that of internal mammary artery and superior to that of saphenous vein. ${ }^{5}$

\section{Conclusion}

We report a case of CABG using RA to repair bilateral COA after ARR in patient with MFS. CABG is feasible to avoid the recurrence of COA. Moreover, CABG using RA graft may be a reasonable selection during reoperation.

\section{Conflicts of interest}

The authors declare no conflicts of interest.

\section{References}

1. Savunen T, Inberg M, Niinikoski J, Rantakokko V, Vänttinen E. Composite graft in annulo-aortic ectasia. Nineteen years' experience without graft inclusion. Eur J Cardiothorac Surg 1996;10:428-432.

2. Meijboom LJ, Nollen GJ, Merchant N, et al. Frequency of coronary ostial aneurysms after aortic root surgery in patients with the Marfan syndrome. Am J Cardiol 2002;89:1135-1138.

3. Kazui T, Yamashita K, Terada H, et al. Late reoperation for proximal aortic and arch complications after previous composite graft replacement in Marfan patients. Ann Thorac Surg 2003;76:1203-1207.

4. Kazuma O, Filip PC, Raphael DG, Hugo V. Giant left coronary ostial aneurysm after modified Bentall procedure in a Marfan patient. Interact Cardiovasc Thorac Surg 2008;7:1164-1166.

5. Gaudino M, Tondi P, Benedetto U, et al. Radial Artery as a Coronary Artery Bypass Conduit: 20-Year Results. J Am Coll Cardiol 2016;68:603-610.

\section{Figure Legends}

Figure 1

Large aneurysms at the bilateral coronary ostial anastomoses.

Figure 2

The both coronary ostia were closed (circle), and the coronary artery bypass (Aorta-Radial artery-Right coronary artery and Aorta- Radial artery-Left main stem) were patent (arrow). 

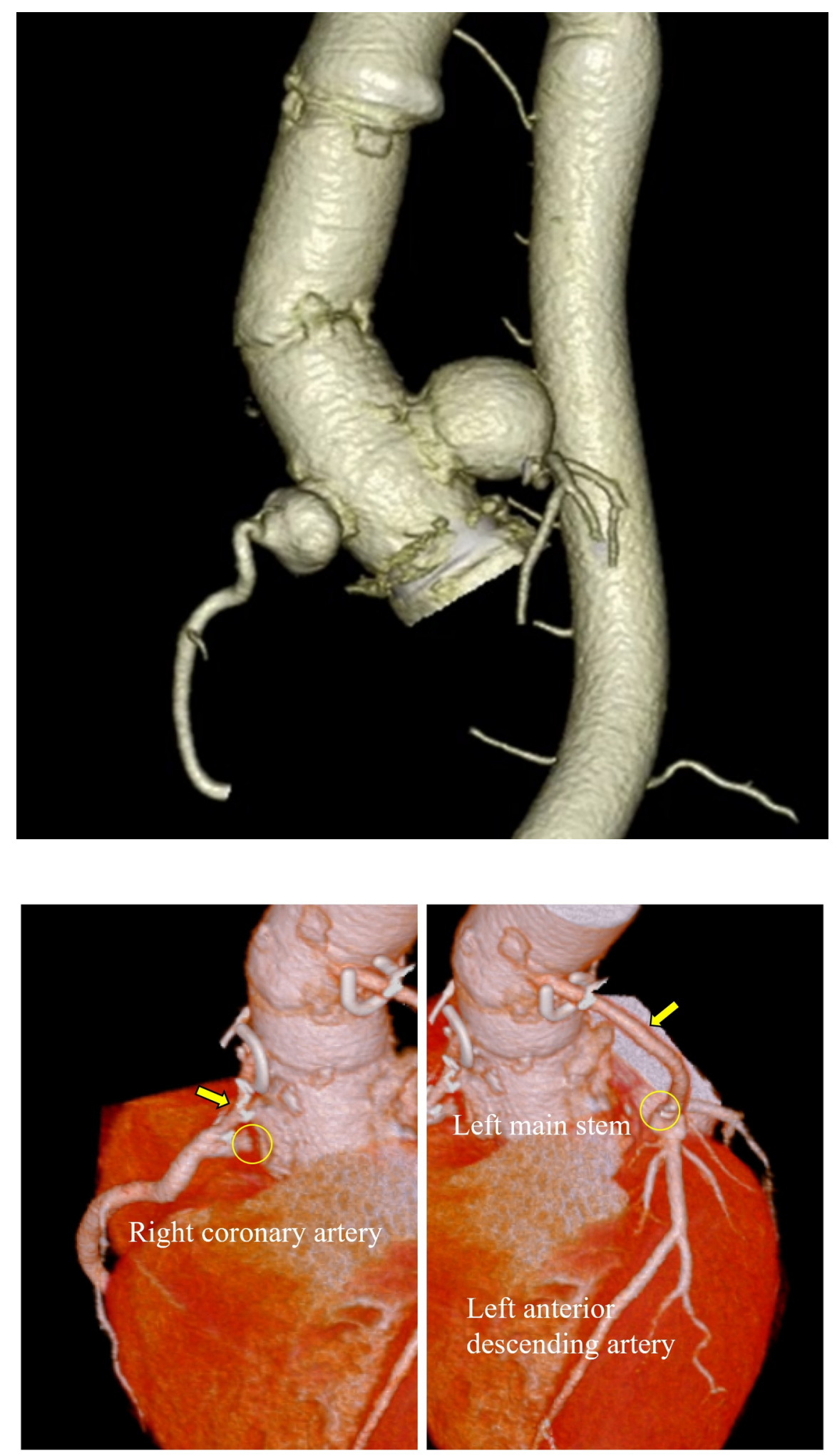